\title{
The representations of the g-Drazin inverse in a Banach algebra
}

\author{
Marjan Sheibani Abdolyousefi (10 \\ Women's University of Semnan (Farzanegan), Semnan, Iran
}

\begin{abstract}
The aim of this paper is to establish an explicit representation of the generalized Drazin inverse $(a+b)^{d}$ under the condition

$$
a b^{2}=0, b a^{2}=0, a^{\pi} b^{\pi}(b a)^{2}=0 .
$$

Furthermore, we apply our results to give some representation of generalized Drazin inverse for a $2 \times 2$ block operator matrix. These extend the results on Drazin inverse of $\mathrm{Bu}, \mathrm{Feng}$ and Bai [Appl. Math. Comput. 218, 10226-10237, 2012] and Dopazo and MartinezSerano [Linear Algebra Appl. 432, 1896-1904, 2010].
\end{abstract}

Mathematics Subject Classification (2020). 15A09, 47L10, 32A65

Keywords. g-Drazin inverse, additive property, perturbation, Banach algebra

\section{Introduction}

Let $\mathcal{A}$ be a complex Banach algebra. An element $a \in \mathcal{A}$ has g-Drazin inverse, i.e., generalized Drazin inverse, if there exists $b \in \mathcal{A}$ such that

$$
b=b a b, a b=b a, a-a^{2} b \in A^{q n i l} .
$$

Here, $\mathcal{A}^{\text {qnil }}=\{a \in \mathcal{A} \mid 1+a x \in \mathcal{A}$ is invertible for every $x \in \operatorname{comm}(a)\}$. We note that $a \in \mathcal{A}^{\text {qnil }} \Leftrightarrow \lim _{n \rightarrow \infty}\left\|a^{n}\right\|^{\frac{1}{n}}=0$. Such $b$, if it exists, is unique, and is called the g-Drazin inverse of $a$, and denote it by $a^{d}$. The g-Drazin inverse in a Banach algebra has various applications in singular differential equations, Markov chains and iterative methods (see $[3,4,11]$ ). New additive results for the g-Drazin inverse in a Banach algebra are presented.

In [2, Theorem 3.1], Bu, Feng and Bai gave some formulas of the Drazin inverse of the sum of two complex matrices under the condition $P Q^{2}=0, Q P^{2}=0$. In Section 2, we extend this result and establish an explicit representation of the generalized Drazin inverse $(a+b)^{d}$ under the condition

$$
a b^{2}=0, b a^{2}=0, a^{\pi} b^{\pi}(b a)^{2}=0,
$$

where $a^{\pi}=1-a a^{d}$ is the spectral idempotent of $a \in \mathcal{A}$.

Email address: sheibani@fgusem.ac.ir

Received: 17.06.2020; Accepted: 08.10.2020 
In Section 3, we consider the g-Drazin inverse of a $2 \times 2$ operator matrix

$$
M=\left(\begin{array}{cc}
A & B \\
C & D
\end{array}\right)
$$

where $A, B, C, D \in \mathcal{L}(X)$. Here, $M$ is a bounded linear operator on $X \oplus X$. This problem has been expensively studied by many authors (see $[1,2,6,7,9]$. We then apply our results to establish new conditions under which $M$ has g-Drazin inverse. This also generalize [7, Theorem 2.2] from the Drazin inverse of complex matrix to the g-Drazin inverse in a Banach algebra under a weaker condition.

Throughout the paper, $\mathcal{A}$ is a complex Banach algebra, $X$ is a Banach space. We use $\mathcal{A}^{d}$ to stands for the set of all g-Drazin invertible $a \in \mathcal{A}$.

Let $x \in \mathcal{A}$ and $p^{2}=p \in \mathcal{A}$. Then we have Pierce matrix decomposition $x=p x p+p x(1-$ $p)+(1-p) x p+(1-p) x(1-p)$. Set $a=p x p, b=p x(1-p), c=(1-p) x p, d=(1-p) x(1-p)$. We use the following matrix version to express the Pierce matrix decomposition of $x$ about the idempotent $p$ :

$$
x=\left(\begin{array}{ll}
a & b \\
c & d
\end{array}\right)_{p}
$$

\section{Additive results}

In this section we establish some additive properties of g-Drazin inverse in Banach algebras. We begin with

Lemma 2.1. Let $\mathcal{A}$ be a Banach algebra, $a, b \in \mathcal{A}^{d}$. Let

$$
x=\left(\begin{array}{ll}
a & 0 \\
c & b
\end{array}\right)_{p} \text { or }\left(\begin{array}{ll}
b & c \\
0 & a
\end{array}\right)_{p} .
$$

Then

$$
x^{d}=\left(\begin{array}{cc}
a^{d} & 0 \\
z & b^{d}
\end{array}\right)_{p} \text { or }\left(\begin{array}{cc}
b^{d} & z \\
0 & a^{d}
\end{array}\right)_{p},
$$

where

$$
z=\left(b^{d}\right)^{2}\left(\sum_{i=0}^{\infty}\left(b^{d}\right)^{i} c a^{i}\right) a^{\pi}+b^{\pi}\left(\sum_{i=0}^{\infty} b^{i} c\left(a^{d}\right)^{i}\right)\left(a^{d}\right)^{2}-b^{d} c a^{d} .
$$

Proof. See [5, Theorem 2.3].

Lemma 2.2. Let $\mathcal{A}$ be a Banach algebra, and let $a, b \in \mathcal{A}^{\text {qnil }}$. If

$$
a b^{2}=0, b a^{2}=0,(b a)^{2}=0,
$$

then $a+b \in \mathcal{A}^{\text {qnil }}$.

Proof. Set

$$
M=\left(\begin{array}{cc}
a^{3}+a^{2} b+a b a & a^{3} b+a b a b \\
a^{2}+a b+b a+b^{2} & a^{2} b+b a b+b^{3}
\end{array}\right) .
$$

Then

$$
\begin{aligned}
M & =\left(\begin{array}{cc}
a^{2} b+a b a & a^{3} b+a b a b \\
0 & a^{2} b+b a b
\end{array}\right)+\left(\begin{array}{cc}
a^{3} & 0 \\
a^{2}+a b+b a+b^{2} & b^{3}
\end{array}\right) \\
& :=G+F .
\end{aligned}
$$

We see that $G^{2}=0, G F G=0$ and $G F^{2}=0$. Moreover, we have

$$
\begin{aligned}
F & =\left(\begin{array}{cc}
a^{3} & 0 \\
a^{2}+b a & 0
\end{array}\right)+\left(\begin{array}{cc}
0 & 0 \\
b^{2}+a b & b^{3}
\end{array}\right) \\
& :=H+K
\end{aligned}
$$


Since $H, K$ are quasinilpotent and $H K=0$, we see that $F$ is quasinilpotent. Therefore $M$ is quasinilpotent. Obviously, $M=\left(\left(\begin{array}{c}a \\ 1\end{array}\right)(1, b)\right)^{3}$. It is obvious that $(1, b)\left(\begin{array}{c}a \\ 1\end{array}\right)$ is quasinilpotent. This completes the proof.

Lemma 2.3. Let $\mathcal{A}$ be a Banach algebra, and let $a \in \mathcal{A}^{d}, b \in \mathcal{A}^{\text {qnil }}$. If

$$
a b^{2}=0, b a^{2}=0, a^{\pi}(b a)^{2}=0,
$$

then $a+b \in \mathcal{A}^{d}$ and

$$
(a+b)^{d}=a^{d}+\sum_{n=0}^{\infty}\left(a^{d}\right)^{n+2} b(a+b)^{n} a^{\pi} .
$$

Proof. Let $p=a a^{d}$. Then we have the Pierce decomposition relatively to the idempotent $p:$

Moreover,

$$
a=\left(\begin{array}{cc}
a_{1} & 0 \\
0 & a_{2}
\end{array}\right)_{p}, b=\left(\begin{array}{ll}
b_{1} & b_{2} \\
b_{3} & b_{4}
\end{array}\right)_{p} .
$$

$$
a^{d}=\left(\begin{array}{cc}
a_{1}^{-1} & 0 \\
0 & 0
\end{array}\right)_{p} \text { and } a^{\pi}=\left(\begin{array}{cc}
0 & 0 \\
0 & 1-a a^{d}
\end{array}\right)_{p} .
$$

Since $b a^{2}=0$, we see that $b a a^{d}=\left(b a^{2}\right) a^{d}=0$, we see that $b_{1}=b_{3}=0$.

We easily see that $a_{2}=a-a^{2} a^{d} \in((1-p) \mathcal{A}(1-p))^{q n i l}$. Since $b\left(1-a a^{d}\right)=b \in \mathcal{A}^{\text {qnil }}$, it follows by $[8$, Theorem 2.1$]$ that $b_{4}=\left(1-a a^{d}\right) b\left(1-a a^{d}\right) \in \mathcal{A}^{q n i l}$. One easily checks that

$$
a_{2} b_{4}^{2}=0, b_{4} a_{2}^{2}=0,\left(b_{4} a_{2}\right)^{2}=0 .
$$

In light of Lemma $2.2, a_{2}+b_{4} \in((1-p) \mathcal{A}(1-p))^{q n i l}$. Thus $\left(a_{2}+b_{4}\right)^{d}=0$, and so

$$
a+b=\left(\begin{array}{cc}
a_{1} & b_{2} \\
0 & a_{2}+b_{4}
\end{array}\right),
$$

It follows by Lemma 2.1 that

$$
(a+b)^{d}=\left(\begin{array}{cc}
a_{1} & b_{2} \\
0 & a_{2}+b_{4}
\end{array}\right)^{d}=\left(\begin{array}{cc}
a^{d} & z \\
0 & 0
\end{array}\right)_{p},
$$

where $z=\sum_{n=0}^{\infty}\left(a^{d}\right)^{n+2} b_{2}\left(a_{2}+b_{4}\right)^{n}$. Therefore

$$
(a+b)^{d}=a^{d}+\sum_{n=0}^{\infty}\left(a^{d}\right)^{n+2} b(a+b)^{n} a^{\pi},
$$

as asserted.

We are now ready to prove the following.

Theorem 2.4. Let $\mathcal{A}$ be a Banach algebra, and let $a, b \in \mathcal{A}^{d}$. If

$$
a b^{2}=0, b a^{2}=0, a^{\pi} b^{\pi}(b a)^{2}=0,
$$

then $a+b \in \mathcal{A}^{d}$. In this case,

$$
(a+b)^{d}=a^{d}+b^{d}+\sum_{n=0}^{\infty}\left(a^{d}\right)^{n+2} b(a+b)^{n}+\sum_{n=0}^{\infty}\left(b^{d}\right)^{n+2} a(a+b)^{n} .
$$

Proof. Let $q=b b^{d}$. Then we have

$$
b=\left(\begin{array}{cc}
b_{1} & 0 \\
0 & b_{2}
\end{array}\right)_{q}, a=\left(\begin{array}{cc}
a_{1} & a_{2} \\
a_{3} & a_{4}
\end{array}\right)_{q} .
$$


Moreover,

$$
b^{d}=\left(\begin{array}{cc}
b_{1}^{-1} & 0 \\
0 & 0
\end{array}\right)_{q} \text { and } b^{\pi}=\left(\begin{array}{cc}
0 & 0 \\
0 & 1-b b^{d}
\end{array}\right)_{q} .
$$

Since $a b^{2}=0$, we see that $a b^{d}=0$; hence, $a_{1} b_{1}^{-1}=0$ and $a_{3} b_{1}^{-1}=0$. It follows that $a_{1}=a_{3}=0$. Thus

$$
a+b=\left(\begin{array}{cc}
b_{1} & a_{2} \\
0 & a_{4}+b_{2}
\end{array}\right)_{p} .
$$

We easily see that $b_{2}=b-b^{2} b^{d} \in((1-p) \mathcal{A}(1-p))^{q n i l}$. Since $a\left(1-b b^{d}\right)=a \in \mathcal{A}^{d}$, by using Cline's formula, we have $a_{4}=\left(1-b b^{d}\right) a\left(1-b b^{d}\right) \in \mathcal{A}^{d}$.

Since $a b^{2}=0$, we see that $a_{4} b_{2}^{2}=\left(1-b b^{d}\right) a\left(1-b b^{d}\right) b^{2}=0$. Also we have

$$
b_{2} a_{4}^{2}=\left(1-b b^{d}\right) b a\left(1-b b^{d}\right) a\left(1-b b^{d}\right)=\left(1-b b^{d}\right) b a^{2}\left(1-b b^{d}\right)=0 .
$$

As $a^{\pi} b^{\pi}(b a)^{2}=0$, we have

$$
\left(\begin{array}{cc}
a_{1} & a_{2} \\
0 & a_{4}
\end{array}\right)_{q}^{\pi}\left(\begin{array}{cc}
0 & 0 \\
0 & 1-b b^{d}
\end{array}\right)_{q}\left(\left(\begin{array}{cc}
b_{1} & 0 \\
0 & b_{2}
\end{array}\right)_{q}\left(\begin{array}{cc}
a_{1} & a_{2} \\
0 & a_{4}
\end{array}\right)_{q}\right)^{2}=0
$$

and so $a_{4}^{\pi}\left(b_{2} a_{4}\right)^{2}=0$. In light of Lemma 2.3 , we get

$$
\begin{aligned}
\left(a_{4}+b_{2}\right)^{d} & =a_{4}^{d}+\sum_{n=0}^{\infty}\left(a_{4}^{d}\right)^{n+2} b_{2}\left(a_{4}+b_{2}\right)^{n} a_{4}^{\pi} \\
& =a^{d}+\sum_{n=0}^{\infty}\left(a^{d}\right)^{n+2} b(a+b)^{n} .
\end{aligned}
$$

In view of Lemma 2.1, we have

$$
(a+b)^{d}=\left(\begin{array}{cc}
b_{1} & a_{2} \\
0 & a_{4}+b_{2}
\end{array}\right)^{d}=\left(\begin{array}{cc}
b_{1}^{-1} & z \\
0 & \left(a_{4}+b_{2}\right)^{d}
\end{array}\right)_{p},
$$

where

$$
z=\sum_{n=0}^{\infty}\left(b^{d}\right)^{n+2} a_{2}\left(a_{4}+b_{2}\right)^{n}\left(a_{4}+b_{2}\right)^{\pi}-b^{d} a_{2}\left(a_{4}+b_{2}\right)^{d} .
$$

Since $b^{d} a^{2}=\left(b^{d}\right)^{2}\left(b a^{2}\right)=0$ and $b^{d} a=0$, we have $b^{d} a_{2}\left(a_{4}+b_{2}\right)^{d}=0$ and

$$
\begin{aligned}
& \left(b^{d}\right)^{n+2} a_{2}\left(a_{4}+b_{2}\right)^{n}\left(a_{4}+b_{2}\right)^{\pi} \\
= & \left(b^{d}\right)^{n+2} a(a+b)^{n}\left(a^{\pi}-\sum_{n=0}^{\infty}\left(a^{d}\right)^{n+1} b(a+b)^{n}\right) \\
= & \left(b^{d}\right)^{n+2} a(a+b)^{n} .
\end{aligned}
$$

Hence,

$$
z=\sum_{n=0}^{\infty}\left(b^{d}\right)^{n+2} a(a+b)^{n} .
$$

Therefore

$$
(a+b)^{d}=a^{d}+b^{d}+\sum_{n=0}^{\infty}\left(a^{d}\right)^{n+2} b(a+b)^{n}+\sum_{n=0}^{\infty}\left(b^{d}\right)^{n+2} a(a+b)^{n} .
$$

as asserted.

Example 2.5. Let

$$
a=\left(\begin{array}{lll}
0 & 0 & 0 \\
0 & 0 & 1 \\
0 & 0 & 0
\end{array}\right), b=\left(\begin{array}{lll}
0 & 0 & 0 \\
0 & 1 & 0 \\
1 & 0 & 0
\end{array}\right) \in M_{2}(\mathbb{C}) .
$$

Then

$$
a b^{2}=0, b a^{2}=0, a^{\pi} b^{\pi}(b a)^{2}=0
$$


It is obvious by computing that

$$
a b^{2}=0, b a^{2}=0, a^{\pi} b^{\pi}(b a)^{2}=0 .
$$

Also

and by the formula of Theorem 2.4 we have,

$$
a^{d}=\left(\begin{array}{lll}
0 & 0 & 0 \\
0 & 0 & 0 \\
0 & 0 & 0
\end{array}\right), b^{d}=\left(\begin{array}{lll}
0 & 0 & 0 \\
0 & 1 & 0 \\
0 & 0 & 0
\end{array}\right)
$$

$$
(a+b)^{d}=\left(\begin{array}{ccc}
0 & 0 & 0 \\
0 & 1 & 0 \\
0 & 0 & 0
\end{array}\right) .
$$

\section{Block operator matrices}

In this section, we apply our results to establish new conditions under which a $2 \times 2$ operator matrix over Banach spaces has g-Drazin inverse. Let $\mathcal{A}=\mathcal{L}(x)$ and $M=$ $\left(\begin{array}{cc}A & B \\ C & D\end{array}\right) \in M_{2}(\mathcal{A})$. We now derive

Theorem 3.1. Let $A$ and $D$ have g-Drazin inverses. If $A B D=0, C B D=0, B C A=$ $0, D C A=0, B C B C=0$ and $D^{\pi} C B C=0$, then $M \in M_{2}(\mathcal{A})^{d}$. In this case

$$
M^{d}=\left(\begin{array}{cc}
A^{d} & B\left(D^{d}\right)^{2} \\
C\left(A^{d}\right)^{2} & D^{d}
\end{array}\right)+\sum_{n=0}^{\infty}\left(\begin{array}{cc}
B\left(D^{d}\right)^{n+3} C & \left(A^{d}\right)^{n+2} B \\
\left(D^{d}\right)^{n+2} C & C\left(A^{d}\right)^{n+3} B
\end{array}\right) M^{n} .
$$

Proof. Let $M=P+Q$, where $P=\left(\begin{array}{cc}A & 0 \\ C & 0\end{array}\right)$, and $Q=\left(\begin{array}{cc}0 & B \\ 0 & D\end{array}\right)$. Then $P, Q$ have g-Drazin inverses. Moreover, we have

$$
P^{d}=\left(\begin{array}{cc}
A^{d} & 0 \\
C\left(A^{d}\right)^{2} & 0
\end{array}\right), Q^{d}=\left(\begin{array}{cc}
0 & B\left(D^{d}\right)^{2} \\
0 & D^{d}
\end{array}\right) .
$$

Then

Thus, we have

$$
P^{\pi}=\left(\begin{array}{cc}
A^{\pi} & 0 \\
-C A^{d} & I
\end{array}\right), Q^{\pi}=\left(\begin{array}{cc}
I & -B D^{d} \\
0 & D^{\pi}
\end{array}\right)
$$

$$
\begin{gathered}
P Q^{2}=\left(\begin{array}{cc}
0 & A B D \\
0 & C B D
\end{array}\right)=0 \\
Q P^{2}=\left(\begin{array}{cc}
B C A & 0 \\
D C A & 0
\end{array}\right)=0 ; \\
(Q P)^{2}=\left(\begin{array}{cc}
B C B C & 0 \\
D C B C & 0
\end{array}\right) ; \\
P^{\pi} Q^{\pi}=\left(\begin{array}{cc}
A^{\pi} & -A^{\pi} B D^{d} \\
-C A^{d} & C A^{d} B D^{d}+D^{\pi}
\end{array}\right) .
\end{gathered}
$$

It is obvious by computing that $P^{\pi} Q^{\pi}(Q P)^{2}=0$. In light of Theorem 2.4, $M$ has g-Drazin inverse. Moreover, we have

$$
\begin{aligned}
M^{d}= & P^{d}+Q^{d}+\sum_{n=0}^{\infty}\left(P^{d}\right)^{n+2} Q M^{n}+\sum_{n=0}^{\infty}\left(Q^{d}\right)^{n+2} P M^{n} \\
= & \left(\begin{array}{cc}
A^{d} & B\left(D^{d}\right)^{2} \\
C\left(A^{d}\right)^{2} & D^{d}
\end{array}\right)+\sum_{n=0}^{\infty}\left(\begin{array}{cc}
0 & \left(A^{d}\right)^{n+2} B \\
0 & C\left(A^{d}\right)^{n+3} B
\end{array}\right) M^{n} \\
& +\sum_{n=0}^{\infty}\left(\begin{array}{cc}
B\left(D^{d}\right)^{n+3} C & 0 \\
\left(D^{d}\right)^{n+2} C & 0
\end{array}\right) M^{n}
\end{aligned}
$$

This completes the proof. 
Corollary 3.2. Let $A$ and $D$ have $g$-Drazin inverses. If $A B D=0, C B D=0, B C A=$ $0, D C A=0$ and $C B C=0$, then $M \in M_{2}(\mathcal{A})^{d}$. In this case,

$$
M^{d}=\left(\begin{array}{cc}
A^{d} & B\left(D^{d}\right)^{2} \\
C\left(A^{d}\right)^{2} & D^{d}
\end{array}\right)+\sum_{n=0}^{\infty}\left(\begin{array}{cc}
B\left(D^{d}\right)^{n+3} C & \left(A^{d}\right)^{n+2} B \\
\left(D^{d}\right)^{n+2} C & C\left(A^{d}\right)^{n+3} B
\end{array}\right) M^{n} .
$$

Proof. This is obvious by Theorem 3.1.

Theorem 3.3. Let $A$ and $D$ have g-Drazin inverses. If $D C A=0, B C A=0, C B D=$ $0, A B D=0, C B C B=0$ and $A^{\pi} B C B=0$, then $M \in M_{2}(\mathcal{A})^{d}$. In this case.

$$
M^{d}=\left(\begin{array}{cc}
A^{d} & B\left(D^{d}\right)^{2} \\
C\left(A^{d}\right)^{2} & D^{d}
\end{array}\right)+\sum_{n=0}^{\infty}\left(\begin{array}{cc}
B\left(D^{d}\right)^{n+3} C & \left(A^{d}\right)^{n+2} B \\
\left(D^{d}\right)^{n+2} C & C\left(A^{d}\right)^{n+3} B
\end{array}\right) M^{n} .
$$

Proof. By virtue of Theorem 3.1, the matrix $\left(\begin{array}{cc}D & C \\ B & A\end{array}\right)$ has g-Drazin inverse. Moreover, we have

$$
\left(\begin{array}{cc}
A & B \\
C & D
\end{array}\right)^{d}=\left(\begin{array}{cc}
0 & I \\
I & 0
\end{array}\right)\left(\begin{array}{cc}
D & C \\
B & A
\end{array}\right)^{d}\left(\begin{array}{cc}
0 & I \\
I & 0
\end{array}\right) .
$$

Therefore we obtain the result.

Corollary 3.4. Let $A$ and $D$ have g-Drazin inverses. If $D C A=0, B C A=0, C B D=$ $0, A B D=0$ and $B C B=0$, then $M \in M_{2}(\mathcal{A})^{d}$. In this case.

$$
M^{d}=\left(\begin{array}{cc}
A^{d} & B\left(D^{d}\right)^{2} \\
C\left(A^{d}\right)^{2} & D^{d}
\end{array}\right)+\sum_{n=0}^{\infty}\left(\begin{array}{cc}
B\left(D^{d}\right)^{n+3} C & \left(A^{d}\right)^{n+2} B \\
\left(D^{d}\right)^{n+2} C & C\left(A^{d}\right)^{n+3} B
\end{array}\right) M^{n} .
$$

Proof. This is obvious by Theorem 3.3.

Lemma 3.5. Let $P$ and $Q \in \mathcal{A}$ have g-Drazin inverses. If $P Q^{2}=0, P Q P=0$, then $P+Q$ has $g$-Drazin inverse and

$$
\begin{aligned}
(P+Q)^{d}= & Q^{\pi} \sum_{i=0}^{\infty} Q^{i}\left(P^{d}\right)^{i+1}+\sum_{i=0}^{\infty}\left(Q^{d}\right)^{i+1} P^{i} P^{\pi}+Q^{\pi} \sum_{i=0}^{\infty} Q^{i}\left(P^{d}\right)^{i+2} Q \\
& +\sum_{i=0}^{\infty}\left(Q^{d}\right)^{i+3} P^{i+1} P^{\pi} Q-Q^{d} P^{d} Q-\left(Q^{d}\right)^{2} P P^{d} Q .
\end{aligned}
$$

Proof. This is proved as in [10, Theorem 2.1].

In [7, Theorem 2.2], Dopazo and Martinez-Serrano investigated Drazin inverse of a $2 \times 2$ block complex matrix under the condition $B C=0, B D C=0$ and $B D^{2}=0$. We now generalize it to the $\mathrm{g}$-Drazin inverse with a weaker condition.

Theorem 3.6. Let $A$ and $D$ have $g$-Drazin inverses. If $B C A=0, C B C B=0, A^{\pi} B C B=$ $0, B D C=0$ and $B D^{2}=0$, then $M \in M_{2}(\mathcal{A})^{d}$. In this case,

$$
\begin{aligned}
M^{d}= & \sum_{i=0}^{\infty}\left(\begin{array}{cc}
0 & 0 \\
0 & D^{\pi} D^{i}
\end{array}\right)\left(P^{d}\right)^{i+1}\left(\begin{array}{cc}
I & \sum_{n=0}^{\infty}\left(A^{d}\right)^{n+2} B D_{n} D \\
0 & I+\sum_{n=0}^{\infty} C\left(A^{d}\right)^{n+3} B D_{n} D
\end{array}\right) \\
& +\left(\begin{array}{cc}
\Gamma & \Delta \\
\Lambda & \Xi
\end{array}\right),
\end{aligned}
$$

where

$$
\begin{gathered}
P^{d}=\left(\begin{array}{cc}
A^{d}+\sum_{n=0}^{\infty}\left(A^{d}\right)^{n+2} B C_{n} & \sum_{n=0}^{\infty}\left(A^{d}\right)^{n+2} B D_{n} \\
C\left(A^{d}\right)^{2}+\sum_{n=0}^{\infty} C\left(A^{d}\right)^{n+3} B C_{n} & \sum_{n=0}^{\infty} C\left(A^{d}\right)^{n+3} B D_{n}
\end{array}\right), \\
A_{1}=A, B_{1}=B, C_{1}=C, D_{1}=0 ; C_{0}=0 \text { and } D_{0}=1 \\
A_{n+1}=A A_{n}+B C_{n}, B_{n+1}=A B_{n}+B D_{n}, C_{n+1}=C A_{n}, D_{n+1}=C B_{n},
\end{gathered}
$$


and

$$
\begin{aligned}
\Gamma= & A^{\pi}-\sum_{n=0}^{\infty}\left(A^{d}\right)^{n+1} B C_{n} \\
\Delta= & -\sum_{n=0}^{\infty}\left(A^{d}\right)^{n+1} B D_{n}, \\
\Lambda= & \sum_{i=0}^{\infty}\left[( D ^ { d } ) ^ { i + 1 } C _ { i } \left(A^{\pi}-\sum_{n=0}^{\infty}\left(A^{d}\right)^{n+1} B C_{n}\right.\right. \\
& \left.+\left(D^{d}\right)^{i+1} D_{i}\left(-C A^{d}-\sum_{n=0}^{\infty} C\left(A^{d}\right)^{n+2} B C_{n}\right)\right] \\
& \quad+D D^{\pi}\left[-C A^{d}-\sum_{n=0}^{\infty} C\left(A^{d}\right)^{n+2} B C_{n}\right] \\
\Xi= & \sum_{i=0}^{\infty}\left[( D ^ { d } ) ^ { i + 1 } C _ { i } \left(-\sum_{n=0}^{\infty}\left(A^{d}\right)^{n+1} B D_{n}\right.\right. \\
& \left.+\left(D^{d}\right)^{i+1} D_{i}\left(I_{n}-\sum_{n=0}^{\infty} C\left(A^{d}\right)^{n+2} B D_{n}\right)\right] \\
& +\sum_{i=0}^{\infty}\left[( D ^ { d } ) ^ { i + 3 } C _ { i + 1 } \left(-\sum_{n=0}^{\infty}\left(A^{d}\right)^{n+1} B D_{n}\right.\right. \\
& \left.+\left(D^{d}\right)^{i+3} C_{i+1}\left(I-\sum_{n=0}^{\infty} C\left(A^{d}\right)^{n+2} B D_{n}\right) D\right]+\left(D^{d}\right)^{2} C \\
& {\left[-\sum_{n=0}^{\infty}\left(A^{d}\right)^{n+1} B D_{n}-D^{d}\left[I-\sum_{n=0}^{\infty} C\left(A^{d}\right)^{n+2} B D_{n}\right] D\right.} \\
& +D D^{\pi}\left[I-\sum_{n=0}^{\infty} C\left(A^{d}\right)^{n+2} B D_{n}\right] \quad(*)
\end{aligned}
$$

Proof. Obviously, we have $M=P+Q$, where

$$
P=\left(\begin{array}{cc}
A & B \\
C & 0
\end{array}\right), Q=\left(\begin{array}{cc}
0 & 0 \\
0 & D
\end{array}\right) .
$$

Clearly, we see that $Q$ has g-Drazin inverse. Since $B C A=0, C B C B=0$ and $A^{\pi} B C B=0$, it follows by Theorem 3.3 that $P$ has g-Drazin inverse and

$$
P^{d}=\left(\begin{array}{cc}
A^{d} & 0 \\
C\left(A^{d}\right)^{2} & 0
\end{array}\right)+\sum_{n=0}^{\infty}\left(\begin{array}{cc}
0 & \left(A^{d}\right)^{n+2} B \\
0 & C\left(A^{d}\right)^{n+3} B
\end{array}\right) P^{n} .
$$

We directly compute that

$$
\begin{gathered}
P Q P=\left(\begin{array}{cc}
B D C & 0 \\
0 & 0
\end{array}\right)=0 \\
P Q^{2}=\left(\begin{array}{cc}
0 & B D^{2} \\
0 & 0
\end{array}\right)=0 .
\end{gathered}
$$

Write $P^{n}=\left(\begin{array}{cc}A_{n} & B_{n} \\ C_{n} & D_{n}\end{array}\right)$. Then $A_{1}=A, B_{1}=B, C_{1}=C, D_{1}=0$ and

$$
A_{n+1}=A A_{n}+B C_{n}, B_{n+1}=A B_{n}+B D_{n}, C_{n+1}=C A_{n}, D_{n+1}=C B_{n} .
$$

Then

$$
P^{d}=\left(\begin{array}{cc}
A^{d}+\sum_{n=0}^{\infty}\left(A^{d}\right)^{n+2} B C_{n} & \sum_{n=0}^{\infty}\left(A^{d}\right)^{n+2} B D_{n} \\
C\left(A^{d}\right)^{2}+\sum_{n=0}^{\infty} C\left(A^{d}\right)^{n+3} B C_{n} & \sum_{n=0}^{\infty} C\left(A^{d}\right)^{n+3} B D_{n}
\end{array}\right),
$$


and so $P^{\pi}=\left(P_{i j}\right)$, where

$$
\begin{aligned}
& P_{11}=A^{\pi}-\sum_{n=0}^{\infty}\left(A^{d}\right)^{n+1} B C_{n} \\
& P_{12}=-\sum_{n=0}^{\infty}\left(A^{d}\right)^{n+1} B D_{n}, \\
& P_{21}=-C A^{d}-\sum_{n=0}^{\infty} C\left(A^{d}\right)^{n+2} B C_{n}, \\
& P_{22}=I-\sum_{n=0}^{\infty} C\left(A^{d}\right)^{n+2} B D_{n} .
\end{aligned}
$$

According to Lemma 3.5, we have

$$
\begin{aligned}
M= & (P+Q)^{d} \\
= & Q^{\pi} \sum_{i=0}^{\infty} Q^{i}\left(P^{d}\right)^{i+1}+\sum_{i=0}^{\infty}\left(Q^{d}\right)^{i+1} P^{i} P^{\pi}+Q^{\pi} \sum_{i=0}^{\infty} Q^{i}\left(P^{d}\right)^{i+2} Q \\
& +\sum_{i=0}^{\infty}\left(Q^{d}\right)^{i+3} P^{i+1} P^{\pi} Q-Q^{d} P^{d} Q-\left(Q^{d}\right)^{2} P P^{d} Q . \\
= & \sum_{i=1}^{\infty}\left(\begin{array}{cc}
0 & 0 \\
0 & D^{\pi} D^{i}
\end{array}\right)\left(P^{d}\right)^{i+1}\left(\begin{array}{cc}
I & \sum_{n=0}^{\infty}\left(A^{d}\right)^{n+2} B D_{n} D \\
0 & I+\sum_{n=0}^{\infty} C\left(A^{d}\right)^{n+3} B D_{n} D
\end{array}\right) \\
& +\sum_{i=0}^{\infty}\left(Q^{d}\right)^{i+1} P^{i} P^{\pi}+\sum_{i=0}^{\infty}\left(Q^{d}\right)^{i+3} P^{i+1} P^{\pi} Q-Q^{d} P^{d} Q \\
& -\left(Q^{d}\right)^{2} P P^{d} Q+Q^{\pi} P^{d} \\
= & \sum_{i=0}^{\infty}\left(\begin{array}{cc}
0 & 0 \\
0 & D^{\pi} D^{i}
\end{array}\right)\left(P^{d}\right)^{i+1}\left(\begin{array}{cc}
I & \sum_{n=0}^{\infty}\left(A^{d}\right)^{n+2} B D_{n} D \\
0 & I+\sum_{n=0}^{\infty} C\left(A^{d}\right)^{n+3} B D_{n} D
\end{array}\right) \\
& +\left(\begin{array}{cc}
\Gamma & \Delta \\
\Lambda & \Xi
\end{array}\right),
\end{aligned}
$$

where $\Gamma, \Delta, \Lambda$ and $\Xi$ are given as in (*) by direct computation.

Acknowledgment. The author is so grateful to the referees for their careful reading and valuable comments.

\section{References}

[1] J. Benítez, X. Qin and X. Liu, New additive results for the generalized Drazin inverse in a Banach Algebra, Filomat 30 (8), 2289-2294, 2016.

[2] C. Bu, C. Feng and S. Bai, Representations for the Drazin inverses of the sum of two matrices and some block matrices, Appl. Math. Comput. 218, 10226-10237, 2012.

[3] S. Campbell, The Drazin inverse and systems of second order linear differential equations, Linear Multilinear Algebra 14, 195-198, 1983.

[4] S.L. Campbell and C.D. Meyer, Generalized inverses of linear transformations, SIAM, 2009.

[5] N. Castro-González and J.J. Koliha, New additive results for the G-Drazin inverse, Proc. Roy. Soc. Edinburgh. Sect A, 134, 1084-1097, 2004.

[6] C. Deng, D.S. Cvetcović-Ilić and Y. Wei, Some results on the genrealized Derazin inverse of operator matrices, Linear Multilinear Algebra 58, 503-521, 2010.

[7] E. Dopazo and M.F. Martinez-Serrano, Further results on the representation of the Drazin inverse of a $2 \times 2$ block matrix, Linear Algebra Appl., 432, 1896-1904, 2010.

[8] Y. Liao, J. Chen and J. Cui, Cline's formula for the generalized Drazin inverse, Bull. Malays. Math. Sci. Soc. 37, 37-42, 2014.

[9] D. Mosić and D.S. Djordjević, Block representations of the generalized Drazin inverse, Appl. Math. Comput. 331, 200-209, 2018. 
[10] H. Yang and X. Liu, The Drazin inverse of the sum of two matrices and its applications, J. Comput. Applied Math. 235, 1412-1417, 2011.

[11] X. Zhang and G. Chen, The computation of Drazin inverse and its applications in Markov chains, Appl. Math. Comput. 183, 292-300, 2006. 